\title{
Photon transport of the superradiant TeraFERMI THz beamline at the FERMI free-electron laser
}

\section{Cristian Svetina, Nicola Mahne, Lorenzo Raimondi, Andrea Perucchi, Paola Di Pietro, Stefano Lupi, Bernhard Schmidt and Marco Zangrando}

J. Synchrotron Rad. (2016). 23, 106-110

\section{\# IUCr Journals CRYSTALLOGRAPHY JOURNALS ONLINE \\ Copyright (C) International Union of Crystallography \\ Author(s) of this paper may load this reprint on their own web site or institutional repository provided that this cover page is retained. Republication of this article or its storage in electronic databases other than as specified above is not permitted without prior permission in writing from the IUCr. \\ For further information see http://journals.iucr.org/services/authorrights.html}




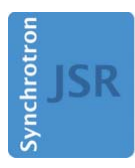

ISSN $1600-5775$

Received 15 July 2015

Accepted 11 November 2015

Edited by E. Plönjes, DESY, Germany

Keywords: TeraFERMI; coherent transition radiation (CTR); FERMI free-electron laser; terahertz beamline; free electron laser (FEL).

\section{Photon transport of the superradiant TeraFERMI THz beamline at the FERMI free-electron laser}

\author{
Cristian Svetina, ${ }^{\mathrm{a}, \mathrm{b} *}$ Nicola Mahne, ${ }^{\mathrm{a}}$ Lorenzo Raimondi, ${ }^{\mathrm{a}}$ Andrea Perucchi, ${ }^{\mathrm{a}, \mathrm{c}}$ \\ Paola Di Pietro, ${ }^{\mathrm{a}, \mathrm{c}}$ Stefano Lupi, ${ }^{\mathrm{c}, \mathrm{d}}$ Bernhard Schmidt $^{\mathrm{e}}$ and Marco Zangrando ${ }^{\mathrm{a}, \mathrm{f}}$ \\ ${ }^{\text {a}}$ Elettra-Sincrotrone Trieste SCpA, SS 14 km, 163.5 in Area Science Park, Trieste 34149, Italy, b Graduate School of \\ Nanotechnology, University of Trieste, Piazzale Europa 1, Trieste 34127, Italy, 'INSTM Udr Trieste-ST, Trieste, Italy, \\ dDipartimento di Fisica, Università 'Sapienza', Ple. A. Moro 5, Roma 00182, Italy, 'Deutsches Elektronen-Synchrotron \\ DESY, Notkestrasse 85, 22607 Hamburg, Germany, and 'IOM-CNR, Laboratorio TASC, SS 14 km, 163.5 in Area Science \\ Park, Trieste 34149, Italy. *Correspondence e-mail: cristian.svetina@elettra.eu
}

TeraFERMI is the new terahertz $(\mathrm{THz})$ beamline for pump-probe studies on the femtosecond time-scale, under construction at the FERMI free-electron laser (FEL) facility in Trieste, Italy. The beamline will take advantage of the coherent radiation emitted by the spent electrons from the FEL undulators, before being dumped. This will result in short, coherent, high-power $\mathrm{THz}$ pulses to be used as a pump beam, in order to modulate structural properties of matter, thereby inducing phase transitions. The TeraFERMI beamline collects $\mathrm{THz}$ radiation in the undulator hall and guides it along a beam pipe which is approximately $30 \mathrm{~m}$ long, extending across the safety hutch and two shielding walls. Here the optical design, which will allow the efficient transport of the emitted $\mathrm{THz}$ radiation in the experimental hall, is presented.

\section{Introduction}

In the last few years, extreme ultra-violet (EUV) and X-ray free-electron lasers (FELs) have become fully operational. These fourth-generation light sources emit brilliant ultra-short pulses with a high degree of coherence and polarization control allowing performance of unprecedented experiments investigating extreme and non-trivial conditions of matter in all its physical states. All these machines are based on the extraction and acceleration of electron bunches and the use of chains of undulators. The linear acceleration sections (namely LINACs) produce naturally terahertz $(\mathrm{THz})$ radiation permitting extension of the working range of operation of these facilities by using dedicated undulators, bending magnets (coherent synchrotron radiation; CSR) or a dedicated target (coherent transition radiation; CTR) (Gensch et al., 2008; Tavella et al., 2011; Stojanovic \& Drescher, 2013; Carr et al., 2002; Daranciang et al., 2011; Hoffmann et al., 2011). In particular, these mechanisms overcome the limitations of other standard $\mathrm{THz}$ sources where the maximum power and/ or bandwidth are limited. FERMI is a seeded FEL user facility at Elettra-Sincrotrone Trieste, in Italy, based on a high-gain harmonic generation (HGHG) scheme that provides almost transform-limited radiation with sub-picosecond photon pulses, narrow bandwidth and high intensity delivered to the experimental end-stations (Allaria et al., 2010, 2012, 2013, 2015). It is composed of two undulator chains: FEL-1, already commissioned, covering the wavelength range from $20 \mathrm{~nm}$ up to $100 \mathrm{~nm}$, and FEL-2, currently under development, but available to users, from $4 \mathrm{~nm}$ up to $20 \mathrm{~nm}$. In Table 1 the main FERMI output parameters are shown. After the FEL process, 
Table 1

Main output parameters measured at FERMI during the commissioning of both FEL-1 and FEL-2.

\begin{tabular}{lll}
\hline Parameter & FEL-1 & FEL-2 \\
\hline Electron beam energy $(\mathrm{GeV})$ & $0.9-1.5$ & $0.9-1.5$ \\
Bunch charge $(\mathrm{nC})$ & $0.5-0.8$ & $0.5-0.8$ \\
Peak current $(\mathrm{A})$ & $500-800$ & $500-800$ \\
Wavelength $(\mathrm{nm})$ & $100-20$ & $20-4$ \\
Pulse length r.m.s. (fs) & $30-100$ & $<100$ \\
Spectral width $\Delta \lambda / \lambda$ FWHM & $10^{-3}$ & $10^{-3}$ \\
Central wavelength fluctuation & $<10^{-4}$ & $<10^{-4}$ \\
Energy per pulse $(\mu \mathrm{J})$ & $>100$ & $20-100$ \\
Power fluctuation $(\%)$ & $<25$ & $<50$ \\
Polarization & Linear $(\mathrm{H} / \mathrm{V})-$ & Linear $(\mathrm{H} / \mathrm{V})-$ \\
& circular $(\mathrm{L} / \mathrm{R})$ & circular $(\mathrm{L} / \mathrm{R})$ \\
& &
\end{tabular}

the electron bunch is deflected from its trajectory by means of a set of bending magnets delivering the electrons to the main beam dump (MBD). Right before the MBD, a thin metallic target screen allows exploitation of the CTR process in order to obtain high-intensity polarized light in a wide terahertz range [for details see Perucchi et al. (2013)]. This light will be transported and used for a wide set of experiments by the beamline TeraFERMI.

By making available high-power sub-picosecond $\mathrm{THz}$ pulses, TeraFERMI will be employed to perform nonlinear $\mathrm{THz}$ spectroscopy and pump-probe studies. Thanks to its large bandwidth (extending from 0.3 to $15 \mathrm{THz}$ ) the TeraFERMI beamline will allow coherent control of matter through nonlinear phononics (Subedi et al., 2014). The main idea is to use the resonant excitation of a vibrational mode in order to transiently change the conformation of the lattice thus providing access to new dynamic electronic configurations. With present bench-top technology (Kampfrath et al., 2013) only a small fraction of the existing vibrational modes can be efficiently reached for $\mathrm{THz}$ control of matter. Due to its low repetition rate $(10-50 \mathrm{~Hz})$, the TeraFERMI facility (Perucchi et al., 2013) will allow excitation of materials over the complete phonon spectrum with negligible heating effects.

\section{THz source properties}

As mentioned in the Introduction, the TeraFERMI source is located in the MBD section, where electrons from both FEL-1 and FEL-2 merge into a single line, before being dumped. A couple of meters before the MBD a dedicated vacuum chamber hosts a set of metallic screens that will be inserted into the electron trajectory. When the ultra-relativistic electrons pass through the target, an intense current arises in the metallic screen radiating in both the forward and backward directions. A sketch of the source is shown in Fig. 1. The TeraFERMI source chamber is equipped with two different screens for CTR: either a $5 \mathrm{~cm}$ Si substrate covered

Figure 1 with a $1 \mu \mathrm{m}$-thick Al coating, or a free-standing $1 \mu \mathrm{m}$-thick $\mathrm{Al}$ membrane. A variable slit between two Si-based emitters can also be used as a source for coherent diffraction radiation. A good prediction of the emission properties in both near and far field can be obtained by applying the generalized Ginzburg-Frank formula (Frank \& Ginzburg, 1945) as described by Casalbuoni et al. (2005, 2009). The emitted radiation is expected to be radially polarized with cylindrical symmetry along the propagation axis, and with an effective source size of $r_{\text {eff }}=\gamma \lambda$, where $\gamma$ is the Lorentz factor and $\lambda$ the wavelength of the radiation. The angular divergence is a function of the frequency as well, being larger at low frequencies, up to $14^{\circ}$. The spectral range covered by TeraFERMI is expected to be exceptionally wide: from $0.3 \mathrm{THz}$ up to $15 \mathrm{THz}$, limited only by the divergence of the photon beam and the transmission of the optical elements (viewports, mirrors, filters, polarimeters) with intensities ranging between $10^{2}$ and $10^{4} \mu \mathrm{J}$ with a pulse length of $50 \mathrm{fs}$ and an electron bunch of $500 \mathrm{pC}$.

At $75 \mathrm{~mm}$ from the source a $600 \mu \mathrm{m}$-thick CVD diamond window separates the main chamber, in ultra-high vacuum (UHV), from the rest of the beamline, in low vacuum. The diamond has a high transmission across the whole TeraFERMI frequency range, around $70 \%$, and an almost constant index of refraction, thus any dispersion will be negligible. With its clear aperture of $20 \mathrm{~mm}$, the window will be a natural filter: it will be able to transmit only radiation above $0.3 \mathrm{THz}$ due to the high divergence at lower frequencies. The surfaces are wedges of about $0.5^{\circ}$ in order to maximize the transmission at all the frequencies and avoid propagating multiple backwardforward reflections. The residual tilt due to the wedge will be compensated by the first plane mirror as described in the next section.

\section{Photon transport}

After the window, the first chamber hosts a plane mirror and the first diagnostic section. The diagnostic system is composed of a translation stage with a screen coupled to a visible CCD camera (in air) dedicated to the alignment of the sourcescreen. In addition, an off-axis parabolic mirror will collect

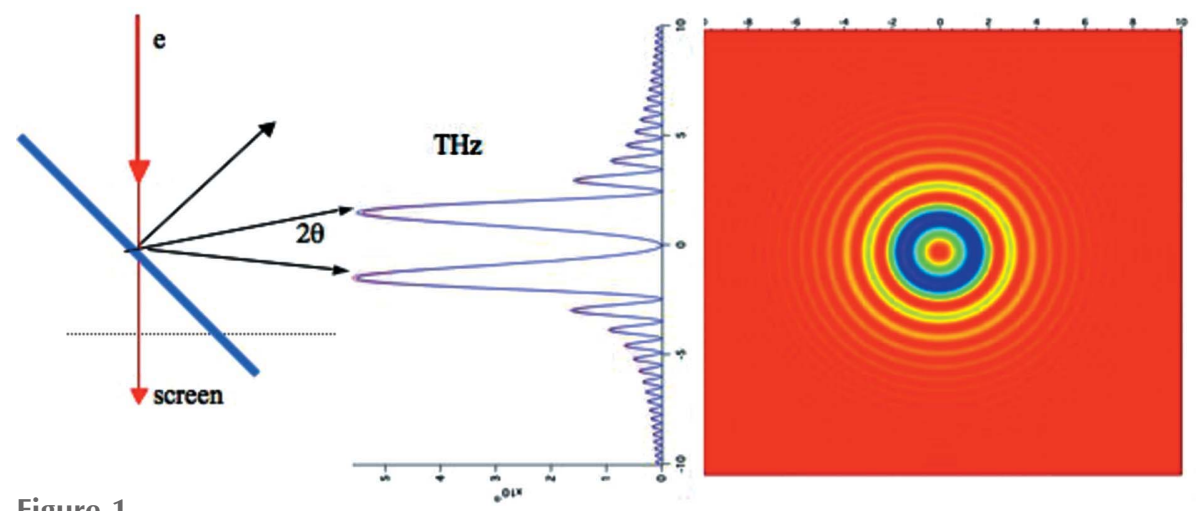

Coherent transition radiation (CTR) mechanism: the relativistic electron bunch interacts with a metallic target and generates broadband terahertz radiation with a cylindrical transverse distribution and high divergence. The slight asymmetry is due to the $45^{\circ}$ incidence angle of the electron bunch over the metallic screen. 
and focus the radiation inside a pyroelectric detector for measuring the emitted intensity in the whole frequency spectrum. The first plane mirror is able to rotate and translate in order to correct residual vertical misalignments of the source, for instance, due to the wedged window. After the first chamber a set of nine other chambers follow, hosting plane and focusing mirrors. All the mirrors work at an incidence angle of $45^{\circ}$ and are $220 \mathrm{~mm}$ wide and $282.8 \mathrm{~mm}$ long (with an elliptical lateral shape) in order to collect as much light as possible, and avoid the diffraction by the aperture. Particular attention has been paid to the finishing of the surfaces. Figure errors and roughness might lead to aberrations of the wavefront and scattered light with a loss of intensity and performance at the sample in general. For this reason we have opted for aluminium substrates gold-coated with a surface accuracy peak-to-valley below $3 \mu \mathrm{m}$ (in clamped conditions) and a roughness below $8 \mathrm{~nm}$ ( $\mathrm{Ra}$, defined as the arithmetic average of the absolute values). A sketch of the layout of the beamline is shown in Fig. 2 and the mirror parameters are reported in Table 2. The optical path has a 'zigzag' pattern in order to fulfil the radiation safety regulations transporting the light from the undulator hall to the experimental hall, passing through the safety hutch. The adopted scheme employs four plane mirrors and six focusing mirrors: the idea is to create intermediate focuses along the beamline in order to limit the beam size within the dimension of the optics. Spherical mirrors have not been considered as an option because the placement of the focal spot is different in the two directions (the focused beam is astigmatic). For this reason we have opted for focusing mirrors with a toroidal shape (manufactured by Kugler), i.e. two different radii of curvature along the tangential and sagittal axis. In this way the beam is stigmatic and limited in its transverse dimension. Two long corrugated beampipes, one in the undulator hall and the other in the safety hutch, will scatter and absorb the undesired off-axis radiation at low frequencies.

One of the by-products of the CTR is the emission of visible light on-axis that will be used for alignment purposes. In fact,
Table 2

Mirror names, distance from the source, type and focal length for the TeraFERMI beamline.

All of the mirrors have a substrate with an elliptical outer shape with semimajor axis of about $141 \mathrm{~mm}$ and semi-minor axis of $100 \mathrm{~mm}$.

\begin{tabular}{llll}
\hline Mirror & $\begin{array}{l}\text { Source distance } \\
(\mathrm{mm})\end{array}$ & Mirror type & $f(\mathrm{~mm})$ \\
\hline PM1 & 480 & Plane & $\infty$ \\
TM1 & 1254 & Toroidal & 680 \\
TM2 & 3900 & Toroidal & 510 \\
TM3 & 5678 & Toroidal & 800 \\
TM4 & 14070 & Toroidal & 3250 \\
PM2 & 15570 & Plane & $\infty$ \\
TM5 & 22822 & Toroidal & 3000 \\
PM3 & 24222 & Plane & $\infty$ \\
PM4 & 30180 & Plane & $\infty$ \\
TM6 & 31180 & Toroidal & 1400 \\
\hline
\end{tabular}

before every mirror, a movable screen can be inserted and seen by a CCD camera while the pointing can be changed by moving the mirror upstream. The overall optical path from the source to the entrance of the TeraFERMI hutch (located in the experimental hall) is about $33 \mathrm{~m}$. Before the hutch, the beam is focused six times and the last focus lies at the beginning of the experimental hutch with a maximum size of around $30 \mathrm{~mm}$ at $0.3 \mathrm{THz}$ and becomes smaller at higher frequencies (for instance it reduces to $12 \mathrm{~mm}$ at $1 \mathrm{THz}$ ).

\section{Beamline spots and transmission}

The design and performance prediction of the whole beamline have been calculated with the Terahertz-Transport code, based on numeric two-dimensional Fourier transform. It is worth noticing that the whole TeraFERMI beamline is in the near-field condition since the far-field approximation is fulfilled when $D>\gamma^{2} \lambda$, thus, considering a representative $\gamma=$ 2300 and $\lambda=1 \mathrm{~mm}$, for very long distances. Starting from the CTR emission the monochromatic beam was propagated through the diamond window, plane and toroidal mirrors and

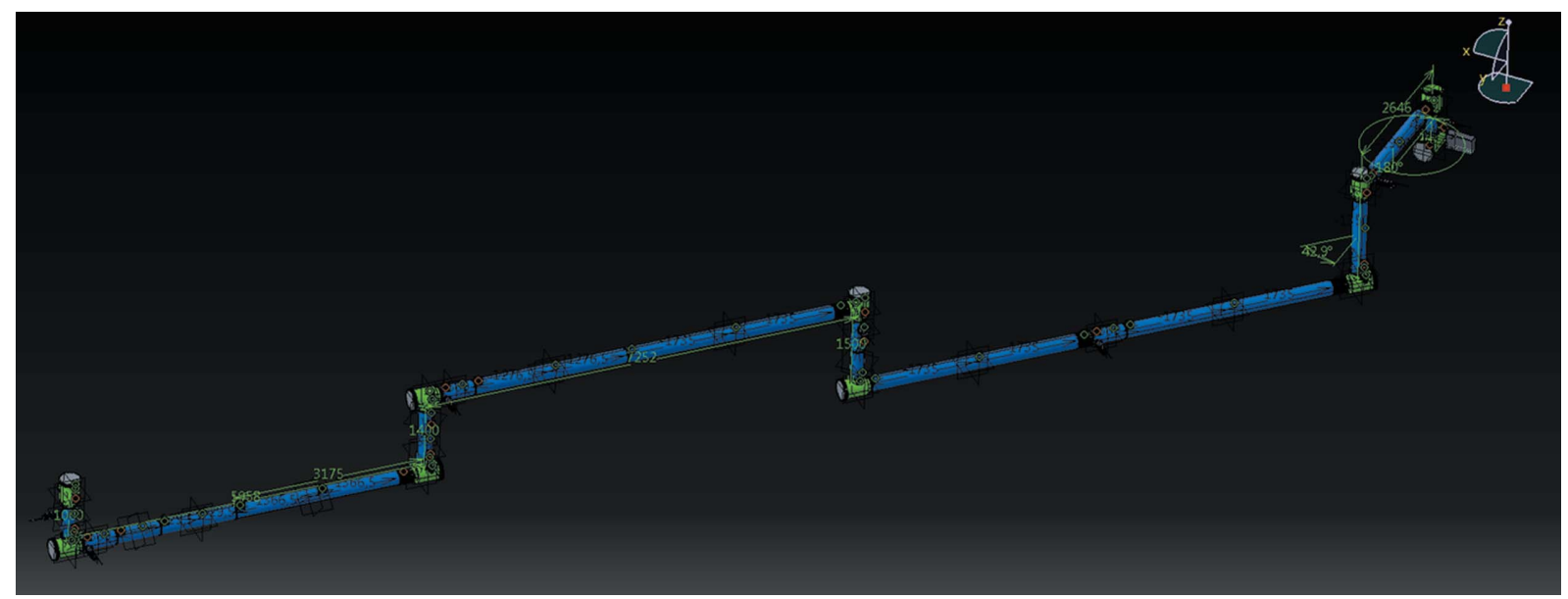

Figure 2

Layout of the TeraFERMI beamline. From right to left: source chamber and plane/toroidal mirrors. The 'zigzag' pattern has been chosen to conform with radiation safety regulations. A set of six toroidal mirrors create intermediate focuses in order to limit the transverse beam dimension while four plane mirrors simply deflect the photon beam. 

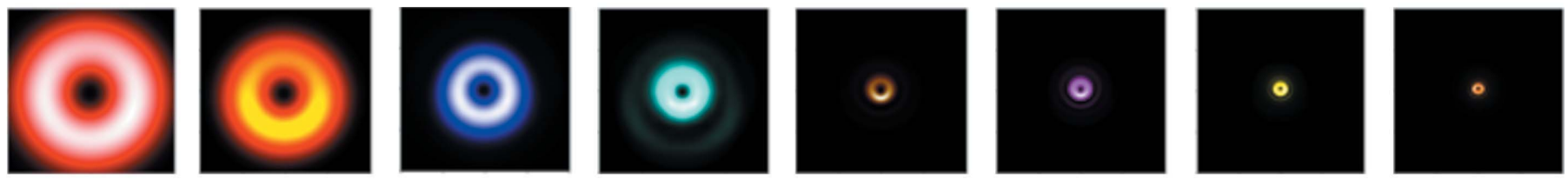

Figure 3

From left to right, simulated spots at $0.3,0.4,0.6,1,3,5,10$ and $15 \mathrm{THz}$ at the entrance of the TeraFERMI hutch in the FERMI experimental hall. The size of each square image is $4 \mathrm{~mm}$ and the spot size dependence on the radiation frequency is evident.

apertures until the TeraFERMI hutch. In this way it was possible to evaluate the focused spot size and transmission of the beamline at the various frequencies. An example of the simulation results in the whole working range is shown in Fig. 3. These calculations have been performed for the whole spectrum and then combined in order to evaluate the overall spot at the entrance of the end-station.

In Fig. 4 the transmission of the beamline is shown after taking into account the transmission of the diamond window and the presence of the mirrors. It can be seen that the first toroidal mirror (blue line) is the major cause of the decrease of the total transmission due to finite acceptance. Nevertheless, it is high enough in the whole frequency range from $83.5 \%$ (at $0.3 \mathrm{THz}$ ) to $95.3 \%$ (at $15 \mathrm{THz}$ ).

\section{Refocusing section and experimental set-up}

As previously mentioned, at the entrance of the TeraFERMI hutch the beam is focused with a maximum dimension of about $30 \mathrm{~mm}$ at $0.3 \mathrm{THz}$. This beam has to be filtered, polarized, split, eventually recombined and, most importantly, focused as much as possible in order to increase the electric field amplitude at the sample. A wide range of experiments planned at TeraFERMI have been considered and a versatile set-up has been adopted. In the following we present the setup that will be used to perform commissioning and pilot experiments. Our aim is to characterize the power and spectral content of the $\mathrm{THz}$ beam as well as its time structure. This goal can be achieved through electro-optic sampling, in the 0.3-

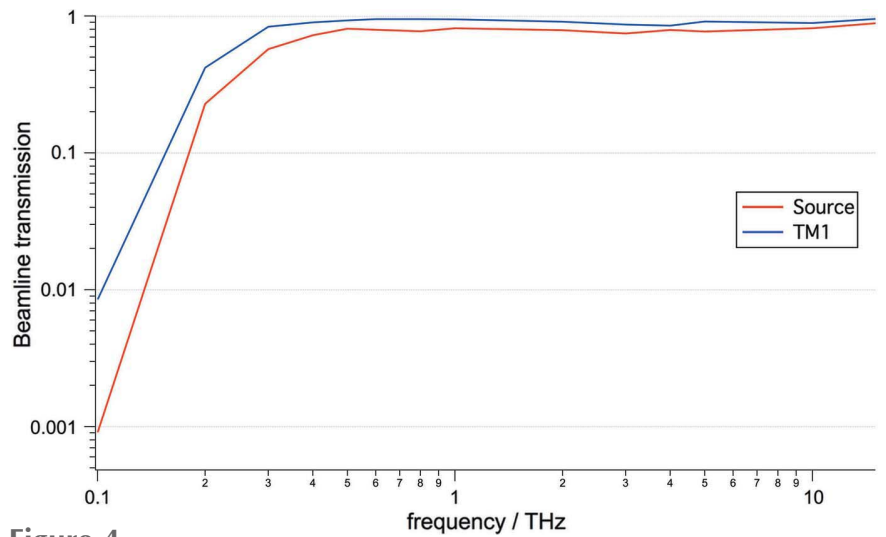

Figure 4

frequency / $\mathrm{THz}$

Transmission due to the presence of the diamond window and the optical elements. The red line is referred to the ideal case of infinitely long mirrors while the blue line is the realistic case with the finite mirrors. It can be seen that the first toroidal mirror is the main cause of the drop in total transmission of the beamline.
$10 \mathrm{THz}$ range (Casalbuoni et al., 2008). We also wish to perform nonlinear experiments on prototype materials. The scheme presented here allows us to easily switch from electrooptic sampling to $\mathrm{THz}$-pump/Vis-probe experiments with the help of a synchronized laser (either 780 or $1560 \mathrm{~nm}$ ) source. Moreover, $\mathrm{THz}$ nonlinear transmission, i.e. the study of the variations in the transmitted light as a function of the beam intensity, will also be possible. A reference channel allowing self-normalization of the results by taking into account possible fluctuations of the source has been envisaged. The optical layout has been designed as follows (see Fig. 5): the incoming beam is focused by an off-axis parabolic mirror (Par1) that focuses the beam at a distance of $215 \mathrm{~mm}$ while passing through a set of filters and polarimeters. Afterwards it propagates through a beam splitter where part of the radiation is reflected to a second off-axis parabolic mirror (Par2) focusing the radiation into a pyro-detector placed $61.6 \mathrm{~mm}$ downstream, used as an on-line I0 monitor. The fraction of radiation transmitted by the splitter is collected by another off-axis parabolic mirror (Par3) which focuses the terahertz radiation to the sample (hosted inside a dedicated chamber) at a distance of $61.6 \mathrm{~mm}$ where the interaction between radiation and matter will occur. Both Par2 and Par3 will be placed $500 \mathrm{~mm}$ downstream of Par1. The light transmitted by the sample will then be collected by a fourth off-axis parabolic mirror (Par4, $150 \mathrm{~mm}$ downstream of Par3) and focused into a second pyro-detector placed $116 \mathrm{~mm}$ downstream.

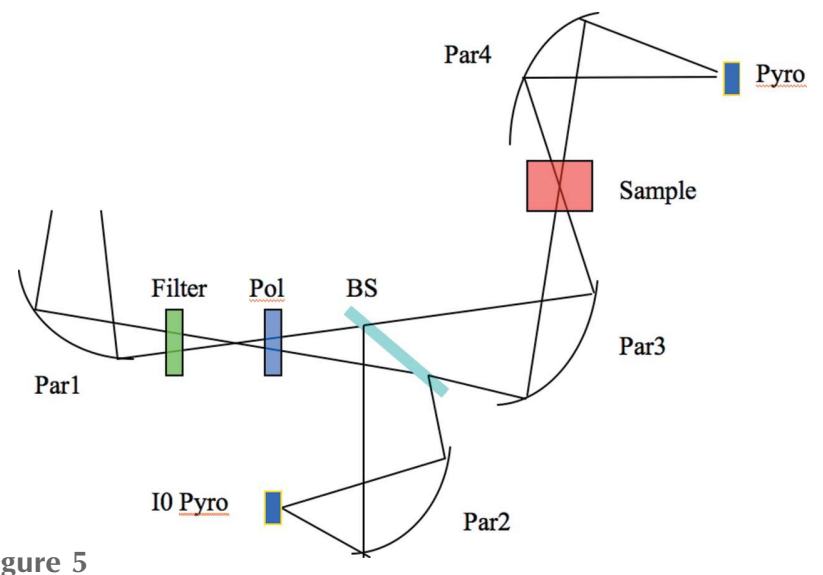

Figure 5

Schematic layout (plan view) of the TeraFERMI refocusing section for the pump-probe pilot experiment. The $\mathrm{THz}$ beam is focused while being filtered and polarized. Then it is split into two beams: one for the on-line measurement of the I0 (with a dedicated mirror, Par2) and one that will be used as a pump pulse. This pulse is focused close to the diffraction limit by another focusing mirror (Par3). The beam is then refocused (Par4) into a pyro-detector which measures the radiation transmitted by the sample. 

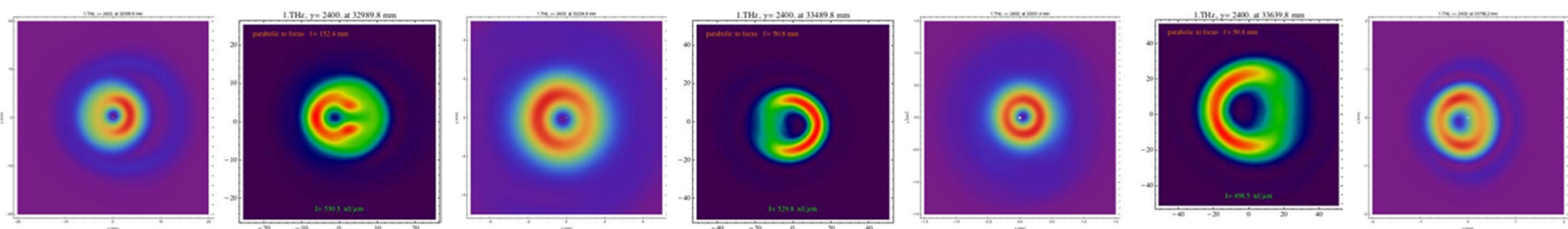

Figure 6

Refocusing section spots simulated with the Terahertz-Transport code at $1 \mathrm{THz}$. From left to right: incoming focused spot from the last toroidal mirror (TM6, FOV = $40 \mathrm{~mm}$ ); footprint over the first parabolic mirror (Par1, FOV = $46 \mathrm{~mm}$ ); intermediate focus due to Par1 (FOV = $10 \mathrm{~mm})$; footprint on the second/third parabolic mirrors (Par2/3, FOV $=100 \mathrm{~mm})$; spot at the I0 pyro-detector and at sample $(\mathrm{FOV}=3 \mathrm{~mm})$; footprint on the last parabolic mirror $($ Par4, FOV $=100 \mathrm{~mm})$; and spot at the pyro-detector in transmission through the sample $(\mathrm{FOV}=4 \mathrm{~mm})$. The spot at the focus is expected to be at the diffraction limit, i.e. around $1 \mathrm{~mm}$ at $1 \mathrm{THz}$.

All the parabolic mirrors work at an incidence angle of $45^{\circ}$. Fig. 6 shows the results of the wavefront propagation simulations over the parabolic mirrors and the relative focal spots simulated with the Terahertz Transport code (http://www. desy.de/ schmidtb/THz-Transport) at $1 \mathrm{THz}$.

\section{Conclusion and outlooks}

By taking advantage of the ultra-relativistic electrons of the FERMI FEL, the coherent transition radiation mechanism will be employed in order to create powerful, coherent, polarized, broadband ultra-short $\mathrm{THz}$ pulses. The radiation will be transported through the undulator hall, the safety hutch and finally to the experimental hall where it will be manipulated and focused according to user needs. The TeraFERMI beamline will allow performance of nonlinear $\mathrm{THz}$ studies and achievement of coherent control of solids through selective excitation of vibrational normal modes. The beamline will work on-line without perturbing the regular operation of the undulator-based FERMI beamlines. TeraFERMI is currently under construction and first light is foreseen by the end of 2015 while the first pilot experiment is planned for the beginning of 2016 .

\section{Acknowledgements}

This work was supported by Italian Ministry of Research (MIUR) program FIRB Futuro in Ricerca Grant No. RBFR10PSK4.

\section{References}

Allaria, E., Appio, R., Badano, L., Barletta, W. A., Bassanese, S., Biedron, S. G., Borga, A., Busetto, E., Castronovo, D., Cinquegrana, P., Cleva, S., Cocco, D., Cornacchia, M., Craievich, P., Cudin, I., D'Auria, G., Dal Forno, M., Danailov, M. B., De Monte, R., De Ninno, G., Delgiusto, P., Demidovich, A., Di Mitri, S., Diviacco, B., Fabris, A., Fabris, R., Fawley, W., Ferianis, M., Ferrari, E., Ferry, S., Froehlich, L., Furlan, P., Gaio, G., Gelmetti, F., Giannessi, L., Giannini, M., Gobessi, R., Ivanov, R., Karantzoulis, E., Lonza, M., Lutman, A., Mahieu, B., Milloch, M., Milton, S. V., Musardo, M., Nikolov, I., Noe, S., Parmigiani, F., Penco, G., Petronio, M., Pivetta, L., Predonzani, M., Rossi, F., Rumiz, L., Salom, A., Scafuri, C., Serpico, C., Sigalotti, P., Spampinati, S., Spezzani, C., Svandrlik, M., Svetina, C., Tazzari, S., Trovo, M., Umer, R., Vascotto, A.,
Veronese, M., Visintini, R., Zaccaria, M., Zangrando, D. \& Zangrando, M. (2012). Nat. Photon. 6, 699-704.

Allaria, E., Badano, L., Bassanese, S., Capotondi, F., Castronovo, D., Cinquegrana, P., Danailov, M. B., D'Auria, G., Demidovich, A., De Monte, R., De Ninno, G., Di Mitri, S., Diviacco, B., Fawley, W. M., Ferianis, M., Ferrari, E., Gaio, G., Gauthier, D., Giannessi, L., Iazzourene, F., Kurdi, G., Mahne, N., Nikolov, I., Parmigiani, F., Penco, G., Raimondi, L., Rebernik, P., Rossi, F., Roussel, E., Scafuri, C., Serpico, C., Sigalotti, P., Spezzani, C., Svandrlik, M., Svetina, C., Trovó, M., Veronese, M., Zangrando, D. \& Zangrando, M. (2015). J. Synchrotron Rad. 22, 485-491.

Allaria, E., Callegari, C., Cocco, D., Fawley, W. M., Kiskinova, M., Masciovecchio, C. \& Parmigiani, F. (2010). New J. Phys. 12, 075002. Allaria, E., Castronovo, D., Cinquegrana, P., Craievich, P., Dal Forno, M., Danailov, M. B., D’Auria, G., Demidovich, A., De Ninno, G., Di Mitri, S., Diviacco, B., Fawley, W. M., Ferianis, M., Ferrari, E., Froehlich, L., Gaio, G., Gauthier, D., Giannessi, L., Ivanov, R., Mahieu, B., Mahne, N., Nikolov, I., Parmigiani, F., Penco, G., Raimondi, L., Scafuri, C., Serpico, C., Sigalotti, P., Spampinati, S., Spezzani, C., Svandrlik, M., Svetina, C., Trovo, M., Veronese, M., Zangrando, D. \& Zangrando, M. (2013). Nat. Photon. 7, 913-918.

Carr, G., Martin, M. C., McKinney, W. R., Jordan, K., Neil, G. R. \& Williams, G. P. (2002). Nature (London), 420, 153-156.

Casalbuoni, S., Schlarb, H., Schmidt, B., Schmüser, P., Steffen, B. \& Winter, A. (2008). Phys. Rev. ST AB, 11, 072802.

Casalbuoni, S., Schmidt, B. \& Schmuser, P. (2005). TESLA Report 2005-15. DESY, Hamburg, Germany.

Casalbuoni, S., Schmidt, B., Schmüser, P., Arsov, V. \& Wesch, S. (2009). Phys. Rev. ST AB, 12, 030705.

Daranciang, D., Goodfellow, J., Fuchs, M., Wen, H., Ghimire, S., Reis, D. A., Loos, H., Fisher, A. S. \& Lindenberg, A. M. (2011). Appl. Phys. Lett. 99, 141117.

Frank, I. M. \& Ginzburg, V. I. (1945). J. Phys. (USSR), 9, 353.

Gensch, M., Bittner, L., Chesnov, A., Delsim-Hashemi, H., Drescher, M., Faatz, B., Feldhaus, J., Fruehling, U., Geloni, G. A., Gerth, Ch., Grimm, O., Hahn, U., Hesse, M., Kapitzki, S., Kocharyan, V., Kozlov, O., Matyushevsky, E., Morozov, N., Petrov, D., Ploenjes, E., Roehling, M., Rossbach, J., Saldin, E. L., Schmidt, B., Schmueser, P., Schneidmiller, E. A., Syresin, E., Willner, A. \& Yurkov, M. V. (2008). Infrared Phys. Technol. 51, 423-425.

Hoffmann, M. C., Schulz, S., Wesch, S., Wunderlich, S., Cavalleri, A. \& Schmidt, B. (2011). Opt. Lett. 36, 4473.

Kampfrath, T., Tanaka, K. \& Nelson, K. A. (2013). Nat. Photon. 7, 680.

Perucchi, A., Di Mitri, S., Penco, G., Allaria, E. \& Lupi, S. (2013). Rev. Sci. Instrum. 84, 022702.

Stojanovic, N. \& Drescher, M. (2013). J. Phys. B, 46, 192001.

Subedi, A., Cavalleri, A. \& Georges, A. (2014). Phys. Rev. B, 89, 220301.

Tavella, F., Stojanovic, N., Geloni, G. \& Gensch, M. (2011). Nat. Photon. 5, 162-165. 